\title{
ABORDAGEM CLÍNICA DAS CEFALÉIAS NA INFÂNCIA
}

\author{
CLINICAL APPROACH TO HEADACHES IN CHILDREN
}

\begin{abstract}
Marco A. Arruda
Pós-graduando do Departamento de Neurologia, Psiquiatria e Psicologia Médica da Faculdade de Medicina de Ribeirão Preto da Universidade de São Paulo.

CorrespondênCIA : R. Casemiro de Abreu, 544 - Ribeirão Preto - São Paulo; CEP 14020-060 - Fones: (016) 636-1640 e 625-7601; Fonefax: (016) 610-6865; E-mail: brheadache@netsite.com.br
\end{abstract}

ARRUDA MA. Abordagem clínica das cefaléias na infância. Medicina, Ribeirão Preto, 30: 449-457, out./dez. 1997.

RESUMO: A cefaléia é um dos sintomas mais freqüentes da infância e apresenta dificuldades diagnósticas específicas. Nesta revisão, analisam-se tais dificuldades e revêem-se os principais tipos de cefaléia, na infância: agudas, crônicas recorrentes e crônicas progressivas; na discussão das cefaléias crônicas, recorrentes, são comentadas as limitações do uso de critérios, diagnósticos de migrânea infantil, propostos pela Sociedade Internacional de Cefaléia, e se propõe um roteiro para o diagnóstico e tratamento da migrânea, na infância.

UNITERMOS: Cefaléia. Enxaqueca. Criança.

\section{INTRODUÇÃO}

A cefaléia é um dos sintomas mais freqüentes da espécie humana ${ }^{(1,2,3)}$ e, na infância, uma das principais queixas, nas clínicas pediátrica e neuropediátrica ${ }^{(4)}$.

Bille, em 1962(5) comprovou parte dessa assertiva através de estudo clássico, em que entrevistou cerca de nove mil crianças da cidade de Upsala, na Suécia. O autor obteve o relato de ao menos um episódio precedente de cefaléia, em $40 \%$ das crianças com sete anos de idade e em $75 \%$ dos adolescentes com quinze anos.

A cefaléia na infância apresenta um largo espectro de causas ${ }^{(6)}$, desde as benignas, como a desencadeada pela febre, até condições clínicas de prognóstico reservado, como a secundária aos tumores e malformações vasculares cerebrais. Entre os dois extremos, encontram-se as cefaléias primárias, como exemplo a migrânea (enxaqueca) e a cefaléia tipo tensional, cada qual com o seu respectivo prognóstico.

A alta frequiência do sintoma na infância, seu amplo espectro etiológico e dados recentes, que alertam para o uso abusivo de analgésicos para cefa- léia nesta faixa etária, justificam a importância do estudo do tema, também, como um problema de saúde pública.

A abordagem das cefaléias na infância compreende não apenas a elucidação da(s) causa(s), mas, sobretudo, a orientação dos pais e da própria criança sobre a sua doença. Essa etapa é de grande importância, uma vez que os pais, muito freqüentemente, suspeitando tão somente de causas graves, só se convencem do contrário após a realização de exames complementares, desnecessários e de alto custo.

O diagnóstico das cefaléias é fundamentalmente clínico, só eventualmente os exames complementares são solicitados para sua confirmação. O médico necessita, portanto, das informações detalhadas do paciente, sobre a dor ${ }^{(4,6 / 11)}$. Daí a maior dificuldade observada no diagnóstico das cefaléias na infância, dadas as limitações encontradas na obtenção de tais informações ${ }^{(4,6,12,13)}$.

A primeira característica clínica, a ser considerada para a definição etiológica das cefaléias, é o aspecto temporal. As cefaléias podem ser divididas em três principais categorias diagnósticas: aguda, crônica recorrente e crônica progressiva (Figura 1). 


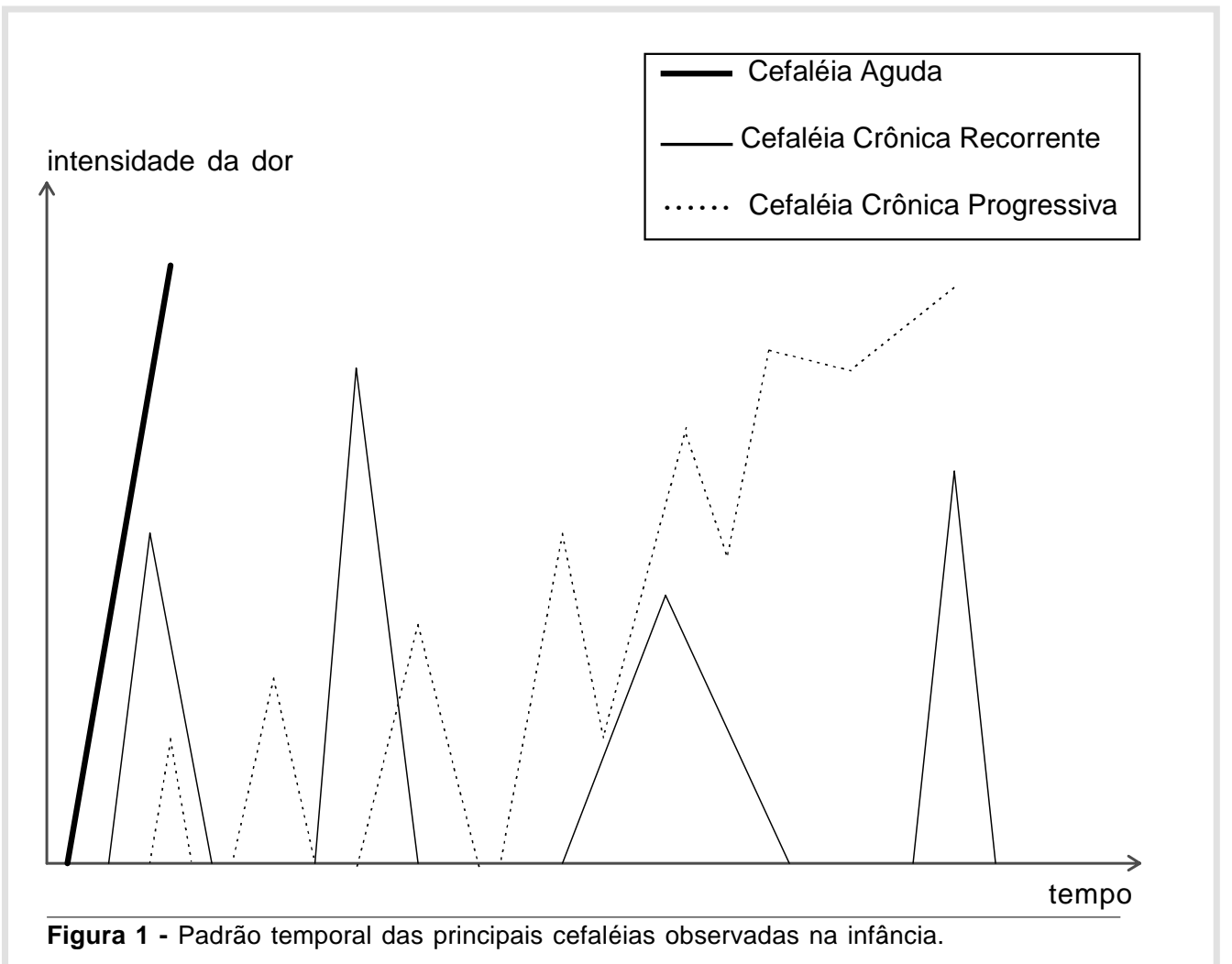

\section{CEFALÉIA AGUDA}

A cefaléia aguda é aquela não sentida anteriormente pela criança e cuja intensidade se agrava rapidamente no decorrer de minutos ou horas. É uma condição clínica, onde a perícia do médico é avaliada, dada a grande incidência de causas graves. Na maioria das vezes, essa cefaléia é provocada por anormalidades estruturais do sistema nervoso ou outros sistemas, o que significa que frequientemente se trata de cefaléia secundária. Daí a grande importância da semiologia geral e neurológica para a elucidação de tais casos.

As causas mais freqüentes estão catalogadas na Tabela I.

\section{Tabela I - Causas de cefaléia aguda na infância}

- Sinusites, otites e mastoidites

- Distúrbios dentários

- Distúrbios oftalmológicos

- Traumatismos cranianos

- Hidrocefalia aguda

- Hemorragia intracranianas

- Meningites e encefalites

- Hipertensão arterial

\subsection{Sinusites, otites, mastoidites}

Habitualmente, provocam cefaléia ou dor facial, de caráter localizado, próximo ao processo infeccioso e inflamatório, e associada a outros sinais e/ou sintomas relacionados. Na maioria das vezes, as radiografias convencionais são suficientes para o diagnóstico das sinusites, mas a tomografia computadorizada pode ser necessária em alguns casos.

\subsection{Distúrbios dentários}

Podem provocar irradiação da dor para áreas adjacentes à cavidade oral, mas esta sempre é o foco de maior intensidade da dor. A inspeção e percussão dos dentes, aliadas aos dados da anamnese (influência da mastigação e da ingestão de líquidos gelados ou quentes sobre a dor, por exemplo) podem definir o diagnóstico.

\subsection{Distúrbios oftalmológicos}

Glaucoma, celulite periorbitária e neurite retrobulbar, por exemplo, podem provocar dor local ou cefaléia generalizada. De observação mais rara na infância, o glaucoma e a neurite retrobulbar podem oferecer dificuldades diagnósticas, necessitando, muitas vezes, da concorrência do oftalmologista para sua resolução. 


\subsection{Traumatismos cranianos}

Podem provocar cefaléia local ou generalizada. Em crianças pequenas, podem faltar informações a respeito do trauma, sendo fundamental a inspeção e palpação do crânio à busca de sinais que confirmem o evento. Lembrar que, em crianças predispostas à migrânea ou com ela já manifesta, é comum a ocorrência ou o agravamento dos ataques após traumas cranianos, mesmo de discreta intensidade. Nesse caso, a presença de vômitos, sonolência e cefaléia de grande intensidade pode confundir o diagnóstico diferencial com as complicações dos traumatismos cranioencefálicos, e a tomografia computadorizada deve ser realizada.

\subsection{Hidrocefalia aguda}

Desencadeia cefaléia de características semelhantes às da hipertensão intracraniana, com nítida exacerbação da dor no período noturno e no início da manhã, muitas vezes, provocando o despertar da criança. Uma particularidade é o predomínio dos vômitos sobre a queixa de cefaléia: são abundantes e rebeldes às medicações, requerendo maior atenção dos pais e do próprio médico ${ }^{(14,15)}$. A semiologia neurológica pode auxiliar o diagnóstico, quando presentes papiledema, macrocefalia e/ou sinal do pote rachado. Havendo suspeita de hidrocefalia, impõe-se a realização da tomografia computadorizada de crânio.

\subsection{Hemorragias intracranianas}

Na maioria das vezes, provocam cefaléia de instalação abrupta, intensidade severa, generalizada ou localizada na região nucal, associadas a vômitos, algum grau de comprometimento da consciência, sinais de irritação meníngea, hemorragias retinianas e/ou sinais localizatórios no exame neurológico. Trata-se de emergência médica, onde a rapidez do atendimento, diagnóstico e tratamento são fatores determinantes da evolução do paciente. Para o diagnóstico, é prudente a realização da tomografia craniana antes da punção liquórica, caso esta seja necessária.

\subsection{Meningites, encefalites}

Cursam com cefaléia, generalizada ou localizada, geralmente no segmento craniano caudal, associada a dor na nuca, febre, náuseas e/ou vômitos, comprometimento da consciência e sinais de irritação meníngea. Havendo suspeita dessa condição, a punção liquórica para a confirmação diagnóstica é de caráter urgente.
2.8. Hipertensão arterial (glomerulonefrite difusa aguda, feocromocitoma, coarctação aórtica)

Provoca cefaléia de instalação abrupta e generalizada ou na porção caudal do crânio, quando na vigência de um pico hipertensivo. No feocromocitoma, vem acompanhada de sudorese, palpitações e/ou ansiedade, manifestações decorrentes da descarga anormal de catecolaminas. Em relação ao grau de hipertensão arterial, a literatura não reconhece que aumentos leves ou moderados da pressão arterial provoquem cefaléia crônica ${ }^{(16 / 19)}$.

\subsection{Investigação laboratorial}

Quando as anormalidades, no exame físico geral e/ou neurológico, apontam para a possibilidade de cefaléia secundária, invariavelmente necessitamos de exames complementares para o diagnóstico etiológico. Os exames mais frequientemente solicitados são: a tomografia computadorizada de crânio, as radiografias dos seios da face e das mastóides, o exame do líquido cefalorraquidiano e o hemograma.

\section{CEFALÉIA CRÔNICA RECORRENTE}

Os protótipos dessa cefaléia são a migrânea (enxaqueca) e a cefaléia tipo tensional episódica. São cefaléias crônicas, que ocorrem em ataques.

\subsection{Migrânea}

Entre as cefaléias crônicas, a migrânea é a mais estudada e, provavelmente, a mais freqüentemente observada na infância ${ }^{(4,6,8,20 / 23)}$. Apesar de inúmeras tentativas, ainda não foram identificados marcadores da migrânea, que permitissem o seu diagnóstico laboratorial ${ }^{(4,10)}$. O diagnóstico da migrânea é estabelecido através da presença de sinais e/ou sintomas característicos dos ataques. No entanto, tais sinais e/ou sintomas podem não ser observados pelos pais ou relatados pelas crianças de menor idade.

$\mathrm{Na}$ tentativa de superar tais dificuldades, diferentes critérios foram propostos para o diagnóstico específico da migrânea na infância ${ }^{(23 / 26)}$.

Em 1988, a Sociedade Internacional de Cefaléia (SIC) propôs uma classificação e critérios diagnósticos para as cefaléias, neuralgias cranianas e dores faciais ${ }^{(27)}$. Para o diagnóstico da migrânea sem aura foram estabelecidos os critérios diagnósticos resumidos na Tabela II. 


\section{Tabela II - Critérios diagnósticos de migrânea (SIC, IHS)}

A. Pelo menos cinco ataques preenchendo os critérios B-D.

B. Crises, perdurando por quatro a setenta e duas horas (não tratadas ou tratadas sem sucesso). Em crianças abaixo de quinze anos, as crises podem durar de duas a quarenta e oito horas. Se o paciente adormece e acorda sem migrânea, considera-se que a crise durou até o momento do despertar.

C. A cefaléia tem, no mínimo, duas das seguintes características:

1. localização unilateral;

2. pulsátil;

3. intensidade moderada ou severa (limitando ou impedindo atividades cotidianas);

4. agravada por subir degraus ou por atividade física rotineira similar.

D. Durante a cefaléia há, no mínimo, um dos seguintes sintomas:

1. náuseas e/ou vômitos;

2. fotofobia e fonofobia.

E. Há, no mínimo, um dos itens relacionados a seguir:

1. História e exames físico e neurológico, sugestivos de um dos distúrbios listados nos grupos de 5 a $11\left(^{*}\right)$;

2. História e/ou exame físico e/ou neurológico, sugestivos de tais distúrbios, mas que são afastados por investigação apropriada;

3. Tais distúrbios estão presentes, mas as crises de migrânea não ocorreram, pela primeira vez, em estreita relação temporal com eles.

(*) Grupos de 5-11: 5) Cefaléia associada a traumatismo craniano; 6) Cefaléia associada a distúrbios vasculares; 7) Cefaléia associada a distúrbio intracraniano, não vascular; 8) Cefaléia associada ao uso de substâncias ou à sua supressão; 9) Cefaléia associada a infecção não cefálica; 10) Cefaléia associada a distúrbio metabólico e 11) Cefaléia ou dor facial associada a distúrbio(s) no crânio, pescoço, nos olhos, ouvidos, no nariz, nos seios, dentes, boca ou em outras estruturas da face ou do crânio.

A literatura comprova a boa sensibilidade e a especificidade dos critérios da SIC $^{(2)}$; no entanto, críticas são feitas à duração dos ataques da migrânea na infância, prevista por esses critérios, uma vez que vários estudos apontam para uma duração freqüentemente mais breve que duas horas.

Contrapondo-se aos critérios anteriormente propostos, os da SIC não consideram a história familiar de migrânea, um dado diagnóstico auxiliar.

A migrânea é um distúrbio geneticamente herdado $^{(9,10,11,28,29)}$. Nas diferentes séries publicadas, a frequiência de história familiar positiva de migrânea (familiares em primeiro grau), varia de 63 a $90 \%$ dos pacientes $^{(5,23,30 / 33)}$. Diferentes tipos de herança já foram postulados para a migrânea, entre eles: herança autossômica dominante (Allan, 1930); autossômica recessiva, com penetrância incompleta (Goodel et al., 1954); autossômica dominante, com alta penetrância no sexo feminino (Barolin, 1969) e poligênica e multifatorial (Dalsgaard-Nielsen \& Ulrich, 1973) (apud 29). Para Ziegler ${ }^{(29)}$ no entanto, a existência de várias síndromes migranosas, faz crer que cada uma tenha sua respectiva influência genética.

De encontro a esta direção, destaca-se a recente descoberta de Joutel et al. ${ }^{(34)}$ que identificaram o gene responsável pela migrânea hemiplégica fami- liar no cromossoma 19 , dando início a uma nova fase da investigação dos fatores hereditários, envolvidos nessa doença.

Outra ferramenta, a nos auxiliar no diagnóstico constitui uma série de sinais e sintomas, freqüentemente apresentados por crianças com migrânea, no período intercrítico, ou seja, fora dos ataques de cefaléia. Apesar das várias denominações empregadas na literatura, tais sinais e sintomas podem ser basicamente definidos como distúrbios associados à migrânea na infância ${ }^{(35)}$. Em estudo comparativo entre crianças com migrânea e sem cefaléia, esses distúrbios se mostraram mais freqüentes, de forma estatisticamente significante, nas crianças do primeiro grupo. São eles: cinetose, dores nos membros, dor abdominal recorrente, febre recorrente, sonambulismo, bruxismo, sonilóquio e terror noturno ${ }^{(35)}$.

Ao ser estudada a relação temporal, existente entre as primeiras manifestações de tais distúrbios e os primeiros episódios de migrânea, através da determinação do risco relativo, observou-se que alguns desses distúrbios, caracteristicamente, manifestavam-se antes do início da cefaléia, podendo então serem também considerados fatores de risco da migrânea na infância. São eles: cinetose, dores nos membros, dor abdominal recorrente, febre recorrente, sonambulismo 
e sonilóquio ${ }^{(35)}$. Portanto, dadas as dificuldades inerentes ao diagnóstico da migrânea na infância, três aspectos devem ser considerados na abordagem diagnóstica dessas crianças: as características dos ataques de cefaléia, a presença de uma história familiar de migrânea e de distúrbios associados à migrânea na infância.

Quanto à terapêutica da migrânea na infância devemos analisar, sobretudo, as particularidades do tratamento dos ataques, as indicações da medicação profilática e os potenciais efeitos colaterais das drogas utilizadas.

De forma característica, os ataques de migrânea na infância, geralmente, resolvem-se com o sono. Isso foi bem observado por Prensky(25) que considerou o efeito benéfico do sono sobre a cefaléia como um dos critérios diagnósticos da migrânea na criança.

Em ataques de menor intensidade e incapacidade, recomenda-se que a criança seja colocada em um ambiente silencioso, escuro e bem ventilado, condições estas que facilitam o sono, podendo, assim, resolver a dor. Se estas medidas iniciais não funcionarem ou se o ataque for de maior intensidade e incapacidade, está indicado o uso de medicamentos. Na ausência de vômitos, optamos por um analgésico comum (Acetaminofeno) ou um antinflamatório não hormonal (AINH) (Naproxen ou Diclofenaco Sódico). Se a criança apresenta náusea e/ou vômito podemos utilizar a Metoclopramida, via retal, ou a Domperidona, via oral, seguida após quinze a vinte minutos do AINH (via oral).

Caso essas condutas não abortem o ataque de migrânea, devem ser consideradas as seguintes opções: Diclofenaco VR, Dipirona EV, Clorpromazina SL ou Dexametasona EV. Os derivados ergotamínicos são contra-indicados na infância, dado o risco potencial de efeitos colaterais graves. Os Triptans, atualmente em voga, ainda não contam com pesquisas que assegurem sua eficácia e segurança no tratamento dos ataques da migrânea na infância.

O tratamento profilático da migrânea na infância é sobretudo medicamentoso, vários trabalhos mostram a ineficácia das dietas ou outras medidas não medicamentosas no controle desta cefaléia. No entanto, técnicas de relaxamento (biofeedback) e modificação comportamental podem beneficiar aquelas crianças cujos aspectos emocionais da cefaléia se sobrepõem aos aspectos eminentemente orgânicos.

A Figura 2 mostra, esquematicamente, as opções medicamentosas no tratamento profilático da criança com migrânea. Esta conduta terapêutica é adotada no Ambulatório de Cefaléia na Infância do HCFMRP-USP e baseia-se na revisão da literatura e na experiência clínica acumulada nos oito anos de funcionamento deste ambulatório.

A posologia das drogas aqui mencionadas bem como seus potenciais efeitos colaterais encontram-se relacionadas na Tabela III.

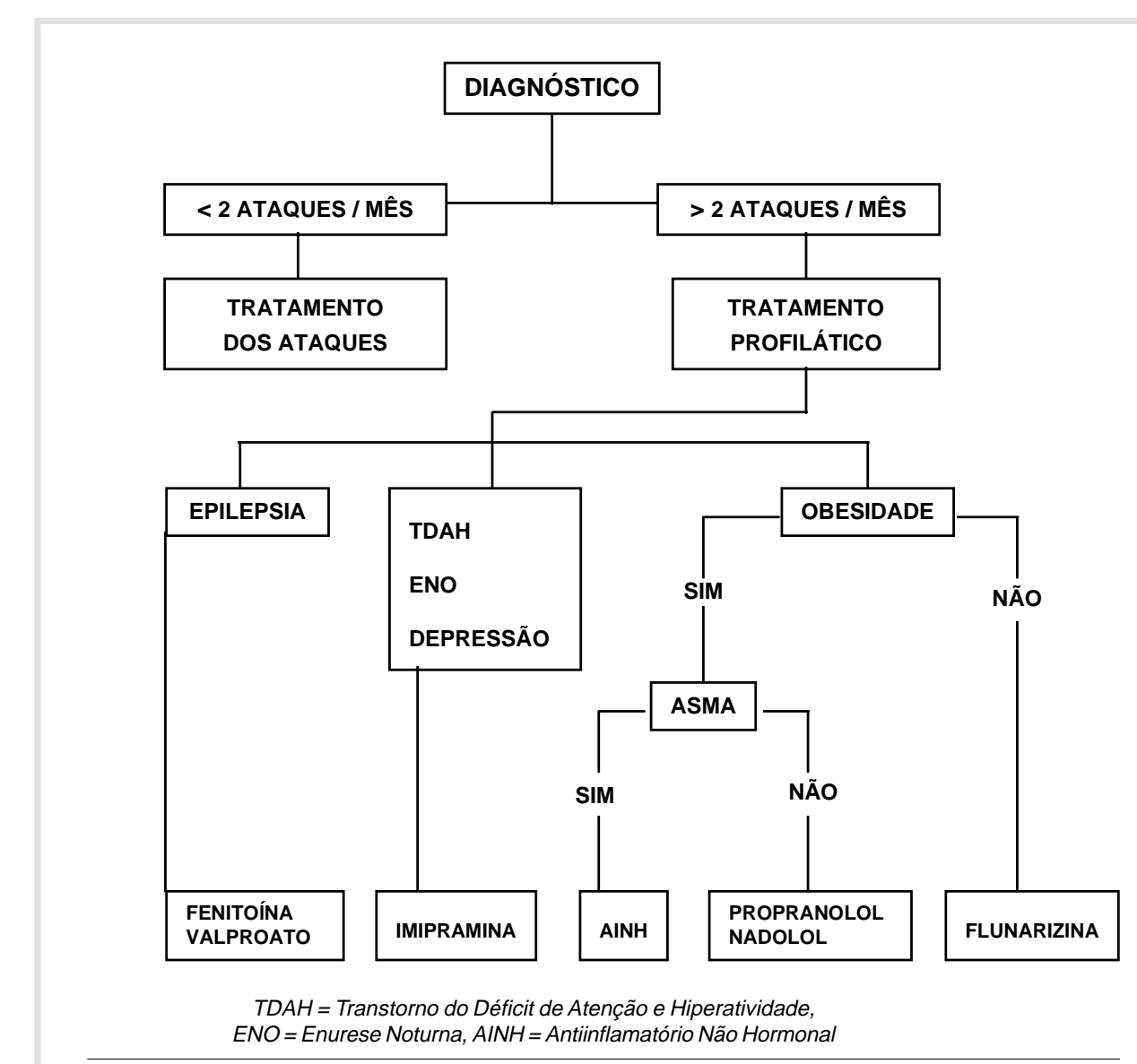

Figura 2 - Fluxograma da conduta terapêutica na migrânea na infância. 
Tabela III - Drogas utilizadas no tratamento profilático e dos ataques de migrânea

\begin{tabular}{|c|c|c|c|}
\hline Droga & Via-Dose & Efeitos Colaterais & Nome Comercial \\
\hline Acetaminofeno & $\begin{array}{l}\text { VO } \\
10 \mathrm{mg} / \mathrm{kg} / \text { dose }\end{array}$ & $\begin{array}{l}\text { Hepatotoxicidade na su- } \\
\text { perdosagem }\end{array}$ & $\begin{array}{l}\text { Tylenol } \\
\text { Dôrico }\end{array}$ \\
\hline Naproxen Sódico & $\begin{array}{l}\text { VO } \\
5 \mathrm{mg} / \mathrm{kg} / \mathrm{dose}\end{array}$ & $\begin{array}{l}\text { Distúrbio Gastrintestinal } \\
\text { Erupção Cutânea }\end{array}$ & $\begin{array}{l}\text { Flanax } \\
\text { Naprosyn }\end{array}$ \\
\hline Diclofenaco & $\begin{array}{l}\text { VO - VR } \\
0,5-2 \mathrm{mg} / \mathrm{kg} / \mathrm{dose}\end{array}$ & $\begin{array}{l}\text { Distúrbio Gastrintestinal } \\
\text { Erupção Cutânea }\end{array}$ & $\begin{array}{l}\text { Biofenac gotas } \\
\text { Cataflam supositório infantil }\end{array}$ \\
\hline Metoclopramida & $\begin{array}{l}\text { VR } \\
0,5 \mathrm{mg} / \mathrm{kg} / \text { dose }\end{array}$ & Distúrbio Extrapiramidal & Eucyl supositórios \\
\hline Domperidona & $\begin{array}{l}\text { VO - IM } \\
0,3-0,4 \mathrm{mg} / \mathrm{kg} / \text { dose }\end{array}$ & $\begin{array}{l}\text { Distúrbio Extrapiramidal } \\
\text { Sonolência }\end{array}$ & Motilium \\
\hline Dipirona EV & $\begin{array}{l}\text { EV } \\
6-15 \mathrm{mg} / \mathrm{kg} / \text { dose }\end{array}$ & $\begin{array}{l}\text { Distúrbio Gastrintestinal } \\
\text { Reações Alérgicas }\end{array}$ & Novalgina \\
\hline Clorpromazina SL & $\begin{array}{l}\mathrm{SL} \\
0,5 \mathrm{mg} / \mathrm{kg} / \mathrm{dose}\end{array}$ & $\begin{array}{l}\text { Sedação } \\
\text { Hipotensão Ortostática } \\
\text { Distúrbio Visual } \\
\text { Retenção Urinária } \\
\text { Constipação }\end{array}$ & Amplictil gotas \\
\hline Dexametasona EV & $\begin{array}{l}\text { EV } \\
0,25 \mathrm{mg} / \mathrm{kg} / \text { dose }\end{array}$ & $\begin{array}{l}\text { No uso por } 1-2 \text { dias os } \\
\text { efeitos colaterais são pou- } \\
\text { co observados. }\end{array}$ & Decadron \\
\hline Fenitoína & $\begin{array}{l}\text { VO } \\
5-10 \mathrm{mg} / \mathrm{kg} / \mathrm{dia} \\
2 \text { doses } / \mathrm{dia}\end{array}$ & $\begin{array}{l}\text { Nistagmo, Ataxia } \\
\text { Sedação } \\
\text { Hiperplasia Gengival } \\
\text { Erupção Cutânea }\end{array}$ & $\begin{array}{l}\text { Epelin } \\
\text { Hidantal }\end{array}$ \\
\hline Ácido Valpróico & $\begin{array}{l}\text { VO } \\
15-60 \mathrm{mg} / \mathrm{kg} / \mathrm{dia} \\
2 \text { doses/dia }\end{array}$ & $\begin{array}{l}\text { Distúrbio Hepático } \\
\text { Perda de Cabelos }\end{array}$ & Depakene \\
\hline Imipramina & $\begin{array}{l}\text { VO } \\
\text { Pré-escolar } 10-40 \mathrm{mg} / \mathrm{dia} \\
\text { Escolar } 10-75 \mathrm{mg} / \mathrm{dia}\end{array}$ & $\begin{array}{l}\text { Efeitos Anticolinérgicos } \\
\text { Bloqueio Adrenérgico }\end{array}$ & Tofranil \\
\hline Propranolol & $\begin{array}{l}\text { VO } \\
1,5-3 \mathrm{mg} / \mathrm{kg} / \mathrm{dia} \\
2 \text { doses/dia }\end{array}$ & $\begin{array}{l}\text { Fadiga } \\
\text { Náusea } \\
\text { Hipotensão Arterial } \\
\text { Bradicardia }\end{array}$ & Propranolol \\
\hline Nadolol & $\begin{array}{l}\text { VO } \\
20-40 \mathrm{mg} / \mathrm{dia} \\
1 \text { dose/dia }\end{array}$ & $\begin{array}{l}\text { Hipotensão Arterial } \\
\text { Bradicardia }\end{array}$ & Corgard \\
\hline Flunarizina & $\begin{array}{l}\text { VO } \\
5-10 \mathrm{mg} / \mathrm{dia} \\
1 \text { dose/dia }\end{array}$ & $\begin{array}{l}\text { Aumento do apetite } \\
\text { Sonolência }\end{array}$ & Sibelium \\
\hline Cipro-heptadina & $\begin{array}{l}\text { VO } \\
0,25 \mathrm{mg} / \mathrm{kg} / \mathrm{dia} \\
2 \text { doses/dia }\end{array}$ & $\begin{array}{l}\text { Aumento do apetite } \\
\text { Sonolência }\end{array}$ & Periatin \\
\hline Pizotifeno & $\begin{array}{l}\text { VO } \\
0,5-1 \mathrm{mg} / \mathrm{dia} \\
1-2 \text { doses/dia }\end{array}$ & $\begin{array}{l}\text { Aumento do apetite } \\
\text { Sonolência }\end{array}$ & Sandomigran \\
\hline
\end{tabular}




\subsection{Cefaléia do tipo tensional episódica}

"Os mecanismos exatos da cefaléia do tipo tensional não são conhecidos. A tensão muscular involuntária, física ou mentalmente induzida, é importante. Também importantes são mecanismos puramente psicogênicos"(27). Aqui temos uma situação controversa, referente não apenas aos mecanismos fisiopatológicos, mas, também, a aspectos clínico-epidemiológicos. Para alguns autores, a cefaléia tipo tensional episódica é a mais freqüente da infância ${ }^{(32,36)}$, para outros, no entanto, é menos freqüentemente observada que a migrânea ${ }^{(4,6,25)}$.

Zeigler \& Hassanein ${ }^{(37)}$ analisaram as características da cefaléia de um mil e duzentos pacientes, atendidos numa clínica especializada em cefaléia. Não foram capazes de identificar uma combinação de sintomas que definissem claramente a migrânea e a cefaléia tipo tensional como doenças distintas. Pacientes que apresentam ambas as cefaléias, usualmente, só distinguem os ataques de uma ou de outra, pela intensidade da dor - maior na migrânea — e/ou pela presença de sintomas associados - geralmente, ausentes na cefaléia tipo tensional episódica ${ }^{(38)}$. A possibilidade de que os dois tipos de cefaléias representem graus diferentes de uma mesma doença justifica parte da divergência de opiniões. A divergência se deve, fundamentalmente, às limitações diagnósticas, encontradas na infância, ao considerarmos uma afecção, onde as informações dadas pelo paciente sejam determinantes no diagnóstico diferencial.

Os critérios da SIC para a cefaléia tipo tensional episódica estão resumidos na Tabela IV.

Para ilustrar algumas limitações dos critérios diagnósticos, bem como certas dificuldades encontradas no diagnóstico diferencial da cefaléia do tipo tensional e da migrânea na infância, podemos analisar o caso clínico apresentado a seguir: Uma criança com cefaléia crônica recorrente em aperto, bilateral e de moderada intensidade, com duração dos ataques de duas horas e associada a anorexia e fotofobia. Tal cefaléia seria diagnosticada como tensional episódica de acordo com os critérios da SIC. Se a criança, ao mesmo tempo, referisse que, ao se exercitar, a cefaléia se tornava pulsátil, e que a dor, apesar de bilateral, tinha um reforço unilateral, o diagnóstico ainda seria de uma cefaléia tipo tensional episódica, pelos critérios da IHS. No entanto, dificilmente um cefaliatra experiente deixaria de fazer o diagnóstico e de fazer o tratamento para migrânea. O exemplo ilustra a importância da anamnese em cefaléia, sobretudo na infância, e dimensiona o papel exato dos critérios diagnósticos das cefaléias na atividade clínica diária.

\section{CEFALÉIA CRÔNICA PROGRESSIVA}

Quando uma criança é levada ao neurologista devido a uma cefaléia, muito freqüentemente os pais suspeitam que ela tenha uma doença mais grave, um tumor cerebral, causa relativamente rara de cefaléia na infância ${ }^{(13)}$. No entanto, se a cefaléia tem início recente (há menos de seis meses), com piora progressiva da intensidade e freqüência dos ataques, a hipótese deve ser considerada.

Tabela IV - Critérios diagnósticos da cefaléia tensional episódica (SIC, IHS)

A. Pelo menos dez episódios prévios de dor de cabeça, preenchendo os critérios de B a D, listados a seguir. Número de dias com cefaléia $<180$ / ano (< 15 / mês).

B. Cefaléia, durando de trinta minutos a sete dias.

C. A dor deve ter, pelo menos, duas das seguintes características:

1. caráter de pressão / aperto (não pulsátil);

2. intensidade fraca ou moderada (pode limitar, porém não impedir as atividades).

3. localização bilateral.

4. não é agravada por subir escadas ou por atividades físicas similares.

D. Ambos os itens seguintes:

1. ausência de náuseas ou vômitos (anorexia pode ocorrer).

2. fotofobia e fonofobia estão ausentes, ou uma mas não a outra está presente.

E. Há no mínimo um dos seguintes itens:

1. história e exames físico e neurológico, não sugestivos de um dos distúrbios listados nos grupos 5-11 (*);

2. história e/ou exame físico e/ou neurológico, sugestivos de tais distúrbios, mas que são afastados por investigação apropriada.

3. esses distúrbios estão presentes, mas as crises de migrânea não ocorreram pela primeira vez, em estreita relação temporal com eles.

(*) Vide Tabela II 
Na Tabela V, a seguir, encontram-se relacionadas outras características clínicas que apontam para a possibilidade de uma cefaléia secundária à hipertensão intracraniana.

Tabela V - Manifestações clínicas sugestivas de cefaléia secundária à hipertensão intracraniana

1. predominam os vômitos sobre a própria cefaléia, especialmente se ocorrem preferencialmente no período noturno e início da manhã, podendo, com isso, provocar o despertar da criança;

2. na evolução da cefaléia, surgem sintomas tais como apatia, anorexia, mudança comportamental, parada do crescimento, macrocefalia, inexplicável déficit visual e/ou declínio progressivo e recente do desempenho escolar;

3. existe(m) anormalidade(s) no exame neurológico $-96 \%$ das crianças com cefaléia secundária a tumor cerebral apresentam anormalidades no exame neurológico, antes dos quatro meses a partir do início da cefaléia $)^{(14)}$;

4. coexistem cefaléia e crises epilépticas, especialmente se as manifestações epiléptica e migranosa ocorrem no mesmo ataque;

5. a cefaléia tem características sugestivas de cefaléia em salvas (risco de malformação vascular cerebral);

6. a cefaléia é desencadeada pelo esforço físico ou manobra de Valsalva;

7. sinais e/ou sintomas neurológicos focais se desenvolvem durante a cefaléia ou persistem ou recorrem após o seu término.
Havendo suspeita de uma cefaléia secundária, torna-se mandatória a realização da tomografia computadorizada e/ou da ressonância magnética nuclear, dependendo de aspectos práticos, referentes à realização do exame e do diagnóstico topográfico e etiológico, suspeitos.

Honig \& Charney ${ }^{(14)}$, num estudo em que analisaram a cefaléia de setenta e duas crianças com tumor cerebral, observaram várias das características acima relacionadas e concluíram ser ineficaz o estudo radiográfico convencional. Nas poucas crianças que apresentavam radiografias simples do crânio anormais, o exame neurológico já se encontrava alterado antes da solicitação. Os autores destacam, por fim, a importância do acompanhamento neurológico e dos retornos breves - para exames neurológicos seriados -, em crianças com cefaléia, cujo tempo de evolução seja inferior a seis meses.

As principais causas de hipertensão intracraniana na criança são a hipertensão intracraniana benigna (pseudotumor cerebral), os tumores cerebrais e a hidrocefalia obstrutiva (a literatura não reconhece a hidrocefalia de pressão normal como causa de cefaléia) ${ }^{(27)}$.

Se a criança apresenta cefaléia crônica progressiva, associada a uma cardiopatia congênita ou imunodeficiência, deve ser afastada a possibilidade de um abscesso cerebral, como também deve ser excluída a possibilidade de um hematoma subdural crônico, se presente, o antecedente de traumatismo craniano significante.

A cefaléia crônica progressiva tem como protótipo a cefaléia da hipertensão intracraniana, mas pode, eventualmente, ser observada no curso de uma cefaléia primária (migrânea ou cefaléia tensional).

ARRUDA MA. Clinical approach to headaches in children. Medicina, Ribeirão Preto, 30: 449-457, oct./dec. 1997.

ABSTRACT: Headache is one of the most frequent symptons in children and it has specific diagnostic problems. In this review, there problems are analyzed and main types of headache in children are discussed - acute, chronic recurrent and chronic progressive. In the discussion of chronic recurrent headaches, limitations of diagnostic criteria of the International Society of Headache for child migraine are commented and a diagnostic and therapeutic guides for migraine in children is proposted.

UNITERMS: Headache. Migraine. Child. 


\section{REFERÊNCIAS BIBLIOGRÁFICAS}

1 - LINET MS et al. An epidemiologic study of headache among adolescents and youn adults. JAMA 261: 2211-2216, 1989.

2 - RASMUSSEN BK et al. Epidemiology of headache in a general population - a prevalence study. J Clin Epidemiol 44: 1147-1157, 1991.

3 - NIKIFOROW R. Headache medication habits in northern Finland. Headache 20: 274-278, 1980.

4 - BARLOW CF. Headaches and migraine in childhood, Blackwell Scientific, Oxford, 288 p, 1984.

5 - BILLE B. Migraine in school children. Acta Paediatr 51: 1-151, 1962. Suppl. 136.

6 - HOCKADAY JM. Migraine in childhood, Butterworths, London, 148 p, 1988.

7 - SHINNAR S \& D'SOUZA BJ. The diagnosis and management of headaches in childhood. Pediatr Clin North Am 29: 79-94, 1981.

8 - GASCON GG. Chronic and recurrent headaches in children and adolescents. Pediatr Clin North Am 31: 1027-1051, 1984.

9 - DALESSIO DJ. Wolff's Headache and other head pain. 15th ed, Oxford University Press, Oxford, 434 p, 1987.

10 - RASKIN NH. Headache. 2nd ed., Churchill Livingstone, New York, 396 p, 1988.

11 - LANCE JW. Mechanism and management of headache. 5th. ed, Butterworth-Heinemann, Oxford, 289 p, 1993.

12 - ELSER JM \& WOODY RC. Migraine headache in the infant and young child. Headache 30: 366-368, 1990.

13 - ROTHNER AD. A practical aproach to headaches in adolescents. Pediatr Ann 20: 200-205, 1991.

14 - HONIG PJ \& CHARNEY EB. Children with brain tumor headaches. Am J Dis Child 136: 121-124, 1982.

15 - TAL Y et al. Childhood migraine - a dangerous diagnosis? Acta Paediatr Scand 73: 55-59, 1984.

16 - CHATELLIER G et al. Symptom prevalence in hypertensive patients. Eur Heart J 3: 45-52, 1982. Suppl. C.

17 - KOTTKE TC et al. The relationship of symptoms and blood pressure in a population sample. Int $\mathbf{J}$ Epidemiol 8: 355-359, 1979.

18 - WATERS WE. Headache and blood pressure in the community. BMJ 1: 142-143, 1971.

19 - WEISS NS. Relation of high blood pressure to headache, epistaxis and selected other symptoms. N Engl J Med 287: 631-633, 1972.

20 - SILBERSTEIN SD. Twenty questions about headaches in children and adolescents. Headache 30: 716-724, 1990.
21 - KÖHLER BF. Headaches in children. Ergeb Inn Med Kinderheilkd 53P: 37-71, 1984.

22 - DYKEN PR. Headaches in children. Am Fam Physician 11: 105-111, 1975.

23 - CONGDON PJ \& FORSYTHE WI. Migraine in childhood : a study of 300 children. Dev Med Child Neurol 21: 209-216, 1979.

24 - VAHLQUIST B. Migraine in children. Int Arch Allergy 7: 348-355, 1955.

25 - PRENSKY AL. Migraine and migrainous variants in pediatric patients. Pediatr Clin North Am 23: 461-471, 1976.

26 - DEUBNER DC. An epidemiologic study of migraine and headache in 10-20 years old. Headache 17: 173-180, 1977.

27 - INTERNATIONAL HEADACHE SOCIETY. Classification and diagnostic criteria for headache disorders, cranial neuralgias and facial pain. Cephalalgia 8: 1-96, 1988. Suppl. 7.

28 - GRAHAM JR. Cefaléia: diagnóstico, mecanismos e tratamento. Trad. de Edgard Raffaeli Junior. FontouraWyeth, São Paulo, 45 p, 1977.

29 - ZIEGLER DK. Genetics of migraine. In: VINKEN PJ et al., eds. Headache. Elsevier Science Publishers, Amsterdam, p. 23-30, 1986. Handbook of clinical neurology, v. 48.

30 - HOLGUIN J \& FENICHEL G. Migraine. J Pediatr 70: 290-297, 1967.

31 - PRENSKY AL \& SOMMER D. Diagnosis and treatment of migraine in children. Neurology 29: 506-510, 1979.

32 - JAY GW \& TOMASI LG. Pediatric headaches : a one year retrospective analysis. Headache 21: 5-9, 1980.

33 - SILLANPÄÄ M. Changes in the prevalence of migraine and other headaches during the first seven school years. Headache 23: 15-19, 1983.

34 - JOUTEL A et al. A gene for familial hemiplegic migraine maps to chromossome 19. Nat Gen 5: 40-45, 1993.

35 - ARRUDA MA. Fatores de risco e distúrbios associados à migrânea na infância. Dissertação de Mestrado, Faculdade de Medicina de Ribeirão Preto da USP, Ribeirão Preto, p 1-117, 1994.

36 - ROTHNER AD. Headaches in children : a review. Headache 18: 169-175, 1978.

37 - ZEIGLER AK \& HASSANEIN RS. Migraine muscle contraction headache dichotomy studied by statistical analysis of headache symptoms. In: CLIFFORD ROSE F, ed. Advances in migraine research and therapy, Raven Press, New York, p. 7-11, 1982.

38 - COHEN MJ \& MCARTHUR DL. Classification of migraine and tension headache from a survey of 10,000 headache diaries. Headache 21: 25-29, 1981.

Recebido para publicação em 05/11/97.

Aprovado para publicação em 10/12/97. 